\title{
Motivação de Estudantes Non-Majors em uma Disciplina de Programação
}

\author{
Bianca L. Santana ${ }^{1}$, José Solenir L. Figuerêdo ${ }^{1}$, Roberto A. Bittencourt ${ }^{1}$ \\ ${ }^{1}$ Programa de Pós-Graduação em Computação Aplicada \\ UEFS - Universidade Estadual de Feira de Santana \\ Av. Transnordestina, s/n, Novo Horizonte \\ Feira de Santana - BA, Brasil - 44036-900 \\ \{biancasantana.ls, solenir.figueredo\}@gmail.com, roberto@uefs.br
}

\begin{abstract}
Motivation plays an important role in students' learning process, helping them to face difficulties. This study investigates how a teaching approach designed for non-majors, that makes use of the Scratch environment, the Python programming language, the Turtle library and the JES IDE, influences students' motivation. We found that Scratch contributed to students' motivation, but JES did not show the same performance. In addition, we use a model to derive a set of variables that may trigger, maintain, or decrease motivation.
\end{abstract}

Resumo. A motivação tem um papel importante no processo de aprendizagem dos estudantes, podendo ajudá-los a enfrentar dificuldades. Neste estudo, investigamos como uma abordagem de ensino de programação para non-majors, que faz uso do ambiente Scratch, da linguagem de programação Python com a biblioteca Turtle e com o ambiente de desenvolvimento JES, influencia na motivação dos estudantes. Verificamos que o Scratch contribuiu para a motivação dos estudantes, enquanto que o JES não mostrou o mesmo desempenho. Além disso, destacamos, a partir de um modelo de motivação, um conjunto de variáveis que podem despertar, manter ou diminuir a motivação.

\section{Introdução}

Programar computadores é uma competência complexa de se aprender por envolver uma variedade de conhecimentos, estratégias e modelos nos diferentes níveis de domínio do problema, do design de solução e do programa propriamente dito [Robins et al. 2003]. Neste contexto, disciplinas de introdução à programação geralmente apresentam altos índices de abandono e reprovação [Watson and Li 2014].

Muitas iniciativas já foram propostas para resolver este problema, alterando currículo, a pedagogia, linguagens de programação e usando ferramentas específicas para apoiar a aprendizagem [Pears et al. 2007]. Apesar disso, estudos evidenciam que as taxas não têm melhorado significativamente ao longo do tempo [Watson and Li 2014]. O insucesso é influenciado por diferentes aspectos, como linguagem utilizada, país, nível escolar, tamanho das turmas e motivação. A motivação afeta, direta ou indiretamente, o desempenho do estudante, com consequências explicitas no processo de aprendizagem. Assim, entender os fatores que estimulam a motivação dos estudantes mostra-se essencial para potencializar o sucesso de cursos de programação. 
Disciplinas de introdução a programação, rotineiramente chamadas de CS1 (Computer Science 1) na literatura, costumam fazer parte das matrizes curriculares de cursos de graduação fora da área de TI, principalmente nas ciências exatas e tecnológicas. Adotamos aqui o termo inglês non-majors para identificar os estudantes que cursam uma disciplina (neste caso, CS1) de uma área diferente da que se graduam. Ensinar programação para non-majors costuma ser ainda mais difícil do que para estudantes de TI, pois os non-majors raramente possuem motivação intrínseca para programar.

Entendemos que as disciplinas de introdução a programação ofertadas para nonmajors podem ser adaptadas para melhor atender este público. Neste sentido, muitos trabalhos já foram desenvolvidos, reformulando currículo, pedagogia, linguagem ou ferramentas para apoiar a prática e a aprendizagem. Várias iniciativas buscam potencializar a motivação e comprometimento dos estudantes [Forte and Guzdial 2005], como cursos focados na habilidade de resolução de problemas, contextualizados para a área do estudante, utilizando as linguagens Logo e Python, utilizando ferramentas como Scratch e Greenfoot, ou utilizando mídias e a ferramenta Jython Environment for Students (JES).

A motivação já vem sendo apontada como um fator importante na aprendizagem há algum tempo. Muitos trabalhos buscam traçar estratégias motivacionais que possam ser usadas pelos professores para fortalecer o desempenho dos estudantes e reforçar atitudes positivas em relação à aprendizagem. A maioria dos psicólogos e educadores usa a motivação como um termo para descrever aqueles processos que podem $(a)$ despertar e instigar o comportamento; $(b)$ dar direção e propósito ao comportamento; $(c)$ continuar a permitir que o comportamento persista; e $(d)$ levar a escolher ou preferir um determinado comportamento [Wlodkowski 1978]. Segundo Jenkins (2001), a motivação pode ser dividida em quatro categorias principais: a extrínseca, a intrínseca, a social e a competitiva.

Compreendendo a importância da motivação, percebe-se que a abordagem empregada pode contribuir para o aumento ou diminuição da motivação dos estudantes [Jenkins 2001]. Para melhor atuar sobre as diversas dimensões da motivação, Keller (1987), criou o modelo ARCS (Atenção, Relevância, Confiança e Satisfação), que inclui um conjunto de estratégias utilizadas para aumentar o apelo motivacional de materiais instrucionais. O ARCS é fundamentado na teoria expectativa versus valor. A expectativa está ligada à satisfação das necessidades pessoais, enquanto o valor representa a razão pela qual um estudante valoriza o sucesso. O modelo ARCS pode ser utilizado no planejamento de materiais instrucionais de uma disciplina, embora isso não garanta ausência de problemas motivacionais. Visando identificar estes problemas, Keller (1993) projetou um instrumento de medição denominado Instructional Material Motivation Survey (IMMS). Este instrumento consta de 36 declarações sobre o material, gerados a partir das categorias definidas pelo ARCS. Para utilizar o IMMS para avaliar motivação, é necessário adaptá-lo à situação da aplicação.

Este trabalho investiga como uma abordagem de ensino-aprendizagem de programação para non-majors, que faz uso do ambiente Scratch, da linguagem de programação Python e do ambiente de desenvolvimento JES, influencia na motivação dos estudantes. A avaliação é realizada a partir de um questionário adaptado do IMMS ao contexto da abordagem, além de uma avaliação qualitativa. O cenário estudado foi uma turma de Introdução a Ciência da Computação (ICC) para calouros de um curso de engenharia Civil. 


\section{Trabalhos Relacionados}

Jenkins (2001) apresenta um trabalho seminal sobre motivação no processo de aprendizagem de programação, evidenciando o papel de um professor como um motivador. Ele categoriza os tipos de motivação existentes e fornece diretrizes que podem contribuir em seu aumento ou diminuição. Um outro trabalho avalia a motivação de estudantes a partir da experiência vivenciada no Georgia Tech, em Atlanta, onde todos os estudantes cursam uma disciplina de CS1 [Forte and Guzdial 2005]. Os cursos de CS1 foram ofertados em três modalidades diferentes, donde duas foram adaptações para non-majors. Os autores avaliaram a motivação e perceberam que os non-majors aproveitaram mais os cursos adaptados, fazendo uso das habilidades aprendidas fora da sala de aula.

Em muitas situações, aprender a programar requer o desenvolvimento de um conjunto de habilidades e competências aprendidas com a prática. Todavia, para que os estudantes sustentem uma rotina de esforço prático, é necessário que estejam adequadamente motivados. Pensando nisso, um trabalho procurou identificar os problemas de motivação de programação entre estudantes de engenharia [Yacob and Saman 2012]. Os autores verificaram que a "direção clara" e a "punição" são os fatores que mais motivaram os estudantes naquela instituição. Em outro trabalho, investiga-se o papel da ferramenta Scratch como elemento motivador [Salazar et al. 2015]. O estudo foi aplicado com 22 alunos de um curso de Bacharelado em Sistemas de Informação. Verificou-se que o Scratch não é suficiente para aprendizagem de programação em disciplinas de graduação, mas a sua utilização na introdução de conceitos iniciais em conjunto com linguagens de programação tradicionais pode aumentar a motivação dos alunos. Um outro trabalho analisa os jogos como meio de gerar motivação, propondo diretrizes para auxiliar docentes na escolha adequada de jogos para o ensino de programação baseada nas técnicas de engajamento e perfis de jogadores [da Silva et al. 2014]. Os autores forneceram um conjunto de observações que auxiliam os professores na escolha dos jogos. Outro estudo apresenta uma investigação sobre a motivação e o uso de estratégias de aprendizagem, por meio de um estudo de caso com estudantes de um curso de Licenciatura em Computação em disciplinas de programação [Peixoto et al. 2013] .

Observando as diretrizes do modelo ARCS, Hamada (2008) introduz um ambiente integrado web para ensino de teoria da computação, levando em conta as preferências de aprendizagem ativa e colaborativa de estudantes de Engenharia de Computação.

\section{Metodologia}

O presente trabalho foi orientado a partir dos métodos mistos, utilizando uma estratégia concomitante. Os métodos mistos combinam os métodos predeterminados das pesquisas quantitativas com métodos emergentes das qualitativas [Creswell 2010]. O estudo foi realizado com uma turma da disciplina de Introdução a Ciência da computação (ICC) do curso de Engenharia Civil de nossa universidade, ao longo do semestre letivo 2016.1. Escolhemos este curso por oferecer a disciplina no primeiro semestre, para os calouros.

\subsection{Participantes}

A turma teve 40 alunos, todos estudantes calouros de Engenharia Civil. Antes de iniciar a pesquisa, elaboramos um termo de consentimento livre e esclarecido (TCLE), descrevendo justificativas, objetivos e possíveis riscos e benefícios ao participar do estudo, além 
de garantir o anonimato dos participantes. Após lerem o TCLE, restaram 36 participantes, sendo 27 do sexo masculino e 9 do feminino.

\subsection{Organização da disciplina}

A disciplina de ICC tem carga horária de 60 horas, divididas entre 30 horas práticas e 30 teóricas. Duas turmas práticas de 20 alunos são apoiadas pelo professor e por um monitor. A disciplina foi dividida em três unidades, cada uma com uma ferramenta diferente. $\mathrm{Na}$ Tabela 1, é sintetizada a organização da disciplina. A utilização de jogos e mídias procurou oferecer um contexto mais adequado e significativo, dado o perfil dos estudantes: entre 17 e 19 anos, uso de redes sociais por meio de dispositivos móveis e afinidade por jogos.

Tabela 1. Descrição Geral da disciplina de ICC

\begin{tabular}{|l|l|}
\hline \multicolumn{2}{|c|}{ Disciplina de Introdução a Ciência da Computação } \\
\hline Unidade I & Conceitos básicos com a ferramenta Scratch. \\
\hline Unidade II & Programação em Python com a biblioteca Turtle. \\
\hline Unidade III & Programação em Python com mídias através do ambiente de desenvolvimento JES. \\
\hline Local & Sala de aula e laboratório de informática. \\
\hline Participantes & 36 estudantes de Engenharia Civil da UEFS e 4 professores. \\
\hline Monitores & Um estudante de Engenharia da computação. \\
\hline Período & Segundo semestre de 2016. \\
\hline Carga horária & 60 horas (30 horas de aula teórica e 30 horas de aula prática). \\
\hline
\end{tabular}

\subsection{Coleta e análise de dados}

Como instrumentos qualitativos, realizamos observações das aulas teóricas e das práticas laboratoriais, bem como entrevistas semiestruturadas com alguns participantes. Ao longo da pesquisa foram realizadas duas entrevistas, sendo que o guia da segunda entrevista observou as quatro dimensões definidas pelo modelo ARCS. Em relação aos instrumentos quantitativos, fizemos uso de três questionários. Dois destes possibilitaram inferir percepções sobre motivação, tendo sido elaborados a partir dos construtos do IMMS.

$\mathrm{Na}$ análise dos questionários, tabulamos dados e geramos estatísticas descritivas e inferenciais, evidenciando características importantes no tocante à motivação. Em contrapartida, com os dados qualitativos das observações e entrevistas, fizemos uma análise descritiva, por meio do processo de codificação, gerando memorandos e descrição de categorias, as quais foram utilizadas para conceber uma classificação de condições que despertam, mantêm ou diminuem a motivação.

\section{Resultados}

Agrupamos os resultados dos questionários a partir dos conceitos do ARCS de atenção, relevância, confiança e satisfação e os apresentamos a seguir.

Na Figura 1, observamos a representatividade da categoria Atenção relacionada à unidade I, com o Scratch. Por exemplo, a variedade de exercícios e ilustrações ajudou a manter a atenção (A1, concordância parcial ou total de 72\%); Scratch estimulou a criatividade (A4, concordância de 64\%); os estudantes concordaram que aprenderam coisas surpreendentes ou inesperadas (A2, 59\%). O design do ambiente não desagradou aos estudantes (A8, discordância parcial ou total de 66\%). O fato de a abordagem com Scratch captar a atenção dos alunos é de grande importância, já que atenção é um elemento de 


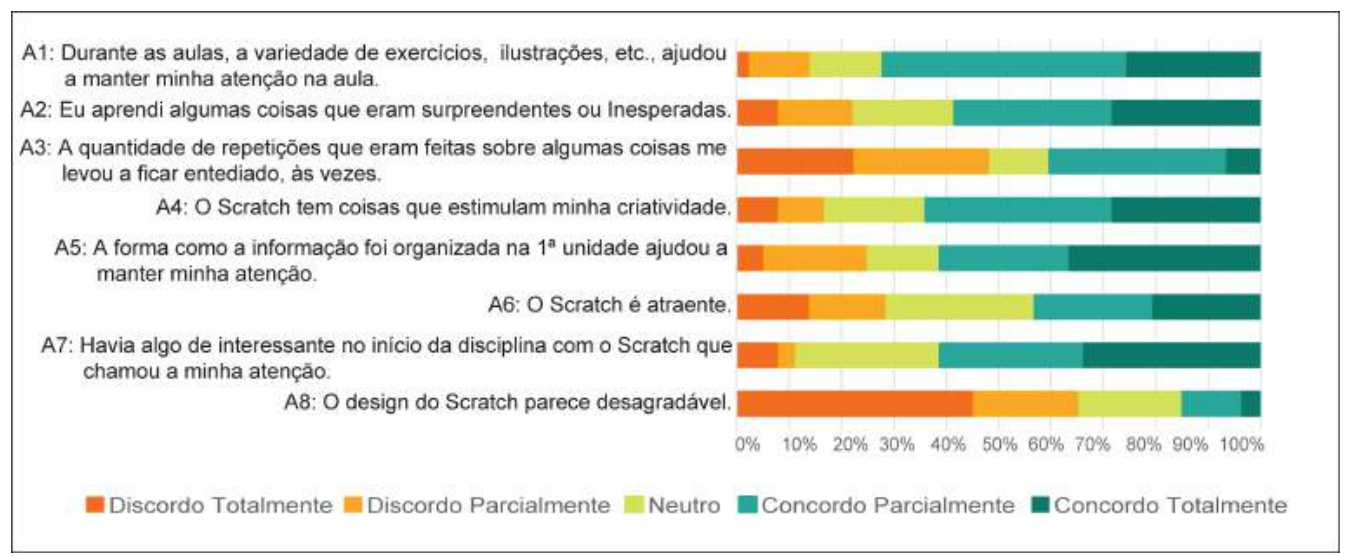

Figura 1. Avaliação da atenção utilizando o ambiente Scratch

motivação e um pré-requisito para aprendizagem [Keller 1987]. Por outro lado, os resultados preliminares na unidade III com o uso do ambiente JES não foram tão positivos. Por exemplo, a variedade de exercícios não ajudou a manter a atenção (A1, concordância de $26 \%$ e e discordância de $43 \%$ ); mais da metade dos alunos concordaram que muitas repetições sobre alguns conteúdos os levaram ao tédio, às vezes (A3, 52\%). As figuras com os resultados da unidade III com o JES não são apresentadas aqui por limitações de espaço.

Na Figura 2, analisamos a categoria Relevância na unidade I com a ferramenta Scratch. Os estudantes perceberam uma relação entre o conteúdo do Scratch e o seu cotidiano (R2, concordância de $61 \%$ ); completar as atividades com sucesso foi importante para os estudantes (R6, concordância de 72\%); e os participantes concordaram o potencial do Scratch para aprender programação (R7, 72\%). Por outro lado, os estudantes não acharam o Scratch significativo para seus interesses (R5, concordância de 9\%). Para avaliar a relevância da ferramenta JES, elaboramos declarações semelhantes. No entanto, ao verificarmos os resultados, notou-se que a percepção de relevância dos estudantes não foi tão expressiva, se comparada aos resultados do Scratch. Por exemplo, os estudantes não acharam os conteúdos com mídia úteis ( $\mathrm{R} 1$, concordância de $24 \%$ ); a relação do conteúdo da terceira unidade com conhecimento prévio também não ficou clara para os estudantes (R2, 47\% de discordância). Entretanto, uma quantidade significativa de estudantes concordou que terminar o projeto com sucesso foi importante (R3, concordância de $51 \%$ e discordância de $12 \%$ ).

Na Figura 3, exibimos os resultados para a categoria Confiança, referente à unidade I com Scratch. O resultado sugere que a ferramenta Scratch despertou a confiança dos estudantes. Por exemplo, os estudantes concordaram que o Scratch proporcionou confiança para aprender o conteúdo $(\mathrm{C} 1,66 \%)$; as aulas introdutórias ajudaram os participantes a compreender o que eles aprenderiam a partir do Scratch (C2, concordância de 63\%); os alunos discordaram de que os exercícios do Scratch foram difíceis (C6, discordância de 66\%); o Scratch não foi difícil de entender (C4, discordância de 71\%). Com relação à avaliação dessa categoria utilizando a ferramenta JES, os resultados iniciais não foram tão positivos, evidenciando que essa ferramenta, em particular, não contribuiu sobremaneira para a confiança dos estudantes. Por exemplo, os estudantes acharam os exercícios difíceis (C3, concordância de 82\%); o número de informações em algumas 


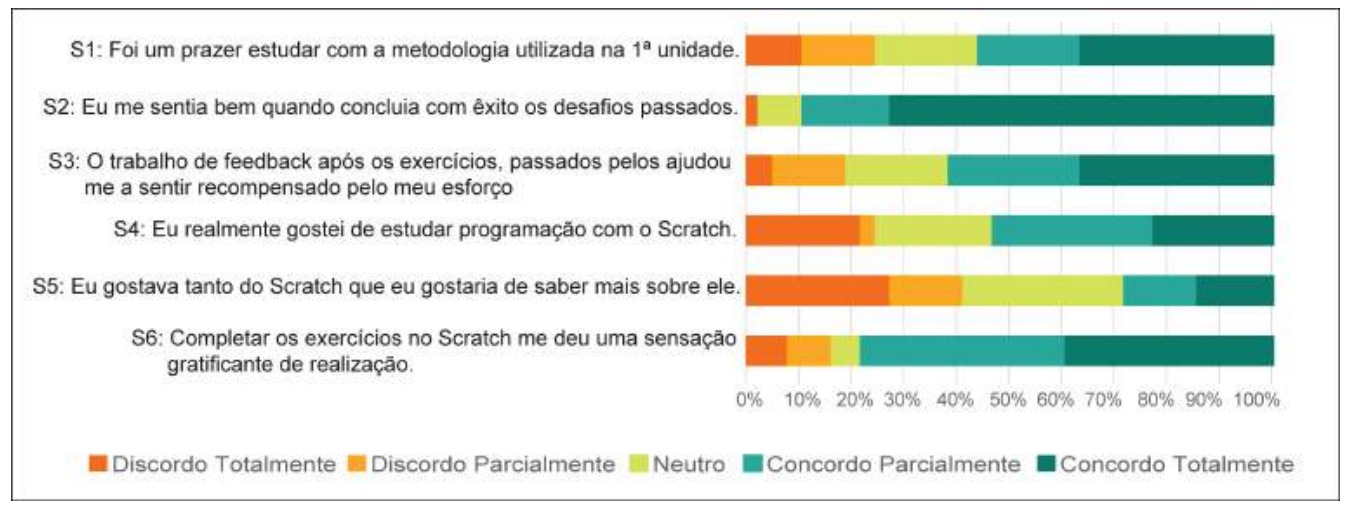

Figura 2. Avaliação da relevância utilizando o ambiente Scratch

aulas atrapalhou no entendimento e na escolha dos pontos principais ( $\mathrm{C} 5$, concordância de 83\%); muitos estudantes discordaram que as aulas introdutórias ajudaram-lhes a sentir confiança sobre o conteúdo a ser aprendido (C6, discordância de 57\%). Apesar disso, os estudantes discordaram de não haver entendido o conteúdo das aulas (C7, 60\%).

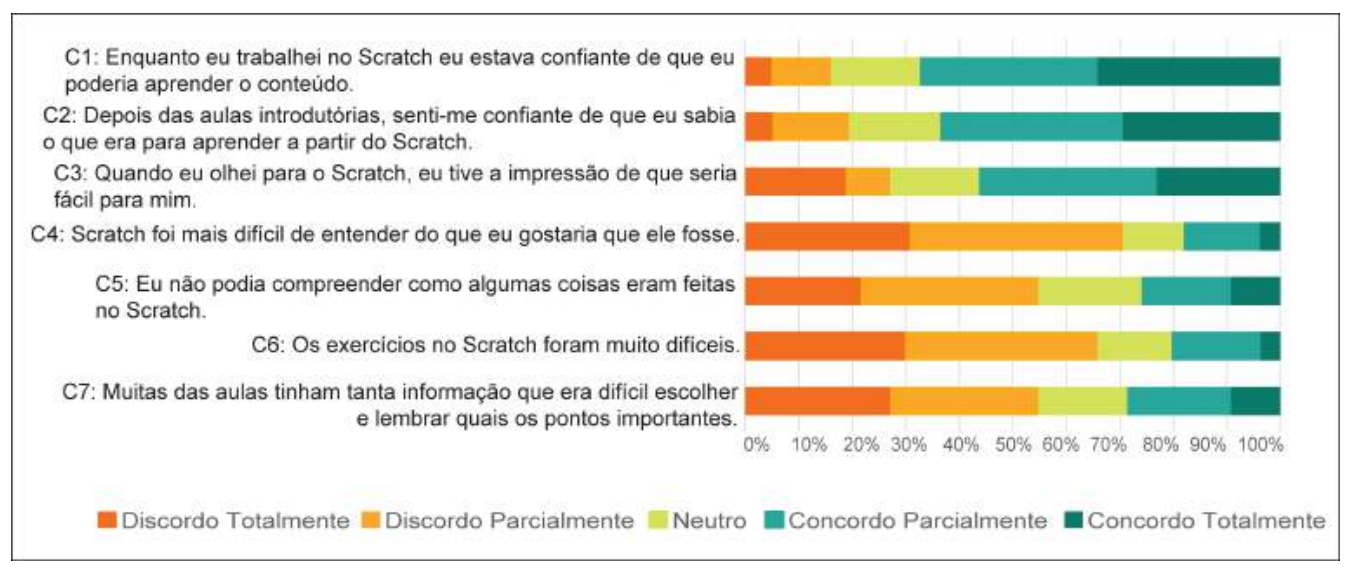

\section{Figura 3. Avaliação da confiança utilizando o ambiente Scratch}

Na Figura 4, exibimos o resultado para a categoria Satisfação ao empregarmos o Scratch. Tivemos evidência de um nível convincente de satisfação, sobretudo em determinadas declarações. Por exemplo, os estudantes concordaram que se sentiam bem ao terminar um desafio com êxito ( $22,89 \%$ ); completar os exercícios proporcionou uma sensação gratificante de realização (S6, concordância de 78\%). No entanto, apesar de o Scratch proporcionar satisfação, uma grande parte dos estudantes discordou de desejar e saber mais sobre ele (S5, discordância de 42\%). Com o JES, a avaliação da satisfação teve alguns resultados positivos. Por exemplo, os estudantes concordaram que a conclusão dos desafios fez eles se sentirem bem (S2, 60\%); completar o projeto deu uma sensação gratificante de realização (S6, concordância de 56\%). Todavia, uma grande parte dos estudantes discordou de ter gostado de estudar manipulação de imagens (S4, discordância de 40\%) e também discordaram de que gostariam saber mais sobre imagens e outras mídias (S5, 74\%). Com a análise dos resultados da satisfação, em especial, nota-se que apesar de os estudantes não terem gostado muito de trabalhar com imagens, a conclusão das atividades com êxito proporcionou grande satisfação. 
A partir dos dados qualitativos coletados por meio das entrevistas e observações, fizemos uma análise, buscando encontrar variáveis relacionadas a estratégias motivacionais, classificadas a partir do modelo definido na Figura 5, ou seja, buscando classificar em fatores que despertam, que mantêm ou que diminuem a motivação.

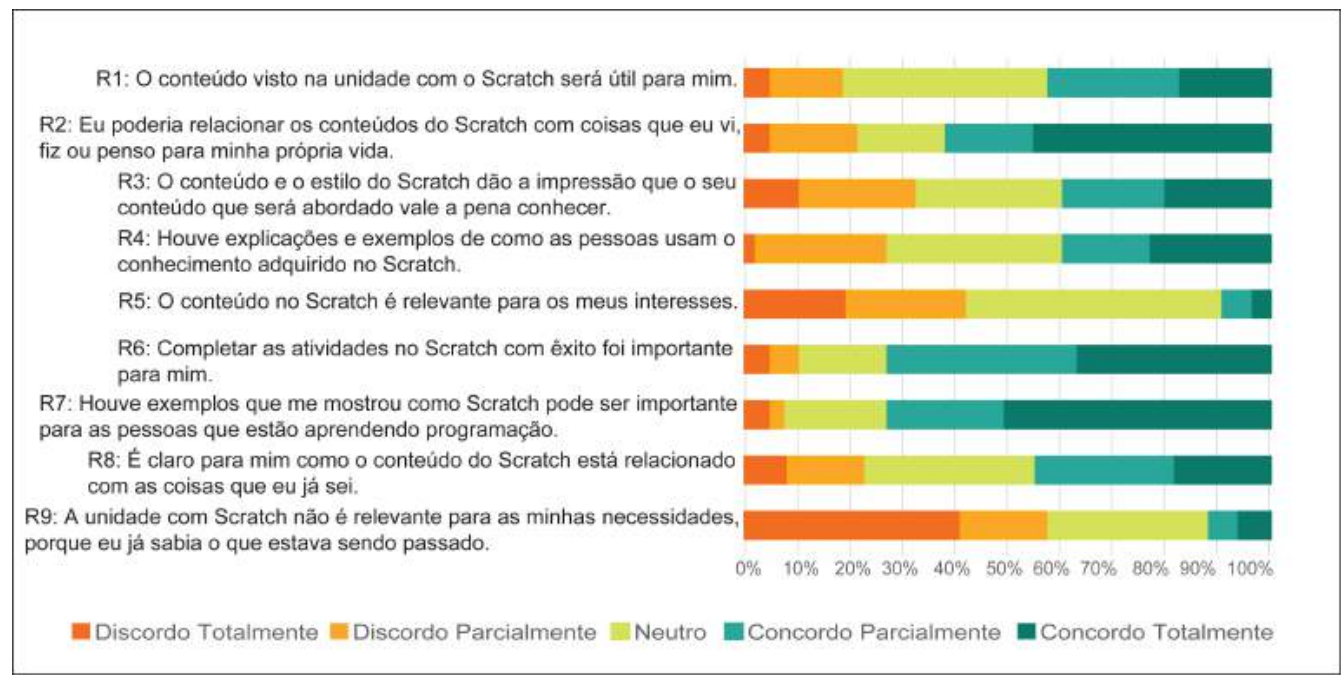

Figura 4. Avaliação da satisfação utilizando o ambiente Scratch

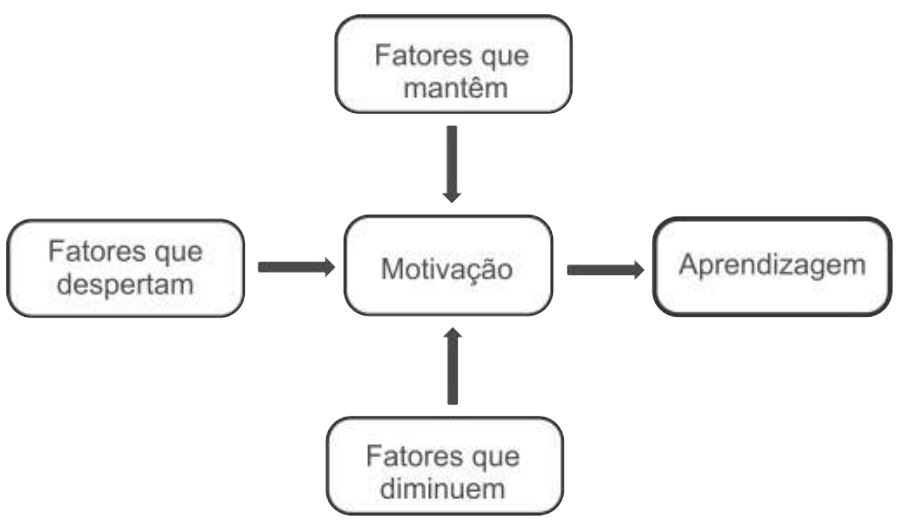

Figura 5. Modelo de classificação das variáveis motivacionais

$\mathrm{Na}$ Tabela 2, listamos as estratégias motivacionais encontradas no decorrer das aulas. Em nosso contexto (ensino de programação), além de encontramos evidências de estratégias selecionadas por Guilloteaux e Dörnyei(2008), encontramos outros fatores relacionados a motivação, que não estão ligados necessariamente à prática do professor, mas à própria abordagem empregada na disciplina. Além disso, muitos fatores que despertaram a motivação também ajudaram a mantê-la.

\section{Discussão}

Percebemos, a partir da abordagem de ensino utilizada, um conjunto de variáveis que podem influenciar positivamente ou negativamente na motivação dos estudantes. Como possíveis variáveis que despertam ou mantêm a motivação, no contexto de ensino de 
Tabela 2. Variáveis motivacionais identificadas

\begin{tabular}{|l|l|l|}
\hline \multicolumn{1}{|c|}{ Despertam a Motivação } & \multicolumn{1}{|c|}{ Mantêm a Motivação } & \multicolumn{1}{|c|}{ Diminuem a Motivação } \\
\hline -Aula prática de programação; & -Aula prática de programação; & -Aula começando com \\
-Experiência anterior com o & -Exercícios valendo nota & certo atraso; \\
conteúdo; & -Compreensão do assunto; & -Didática do professor; \\
-Utilizar Jogos; & -Estudar conteúdo antes da aula; & -Exercícios de programação \\
-Aula interativa; & -Construir os exemplos passo a passo; & feitos em caderno; \\
-Incentivar o aluno a chegar a & -Concluir exercícios com êxito; & -Idioma estrangeiro; \\
resposta sem entregar a resposta & -Utilizar desafios nos exercícios; & -Mudança brusca de um \\
de imediato; & -Utilizar Jogos; & assunto considerado fácil \\
-Influência familiar; & -Scaffolding ou apoio, seja de material & para um mais avançado; \\
-Percepção da utilidade & ou por monitoria; & -Não concluir exercício \\
do assunto; & -Conteúdo ligado ao contexto do estudante; & com êxito; \\
-Gostar de aprender & -Programação individual; & -Professor desencorajando \\
-Atividades com objetivos & -Feedback neutro; & quando de assuntos “difíceis"; \\
bem definidos; & -Feedback do processo; & -Problemas da estrutura física \\
-Despertar a curiosidade; & -Elogio eficaz; & do laboratório; \\
-Estimular autonomia;. & -Incentivar a auto ou correção em pares; & -Sintaxe complicada;. \\
\hline
\end{tabular}

programação, destacamos o feedback neutro e de processo, a utilização de jogos, a existência de scaffolding, a importância das aulas práticas, a promoção da autonomia, exercícios utilizando uma escala gradual de dificuldade e êxito na conclusão dos exercícios. No que concerne às variáveis que diminuem a motivação, evidenciamos a não conclusão de uma atividade, a didática do professor, exercícios de programação feitos no caderno, idioma estrangeiro e mudança brusca da dificuldade de um assunto.

O Scratch e o Python com mídias apresentaram diferentes impactos na motivação. De maneira geral, a primeira unidade obteve avaliações mais positivas que as seguintes.

\section{Scratch}

A escolha do Scratch para a primeira unidade objetivou promover uma introdução mais branda aos conceitos iniciais de programação e potencializar a motivação dos estudantes. Conforme identificado nos dados, a ferramenta exerceu de fato este papel. O Scratch possui um design agradável e o modo como o conteúdo está disposto e foi abordado contribuiu para o aumento da atenção dos estudantes. Além disso, a utilização do Scratch num contexto de criação de jogos estimula a criatividade, o que também ajudou a manter a atenção dos estudantes.

O fato de os estudantes não se sentirem sobrecarregados, nem com dificuldades para realizar as atividades com Scratch, tornou os conceitos iniciais acessíveis. Ao minimizar as dificuldades, a confiança é potencializada. Quanto mais confiante, maior é a persistência e a expectativa de sucesso e, consequentemente, maior é o empenho dos estudantes nas atividades, percepção reforçada nas observações e entrevistas, que indicam que Scratch proporcionou um scaffolding benéfico para a manutenção da motivação.

Por outro lado, o Scratch não passou a sensação de utilidade prática na vida dos estudantes, o que diminui sua relevância percebida (como na típica pergunta: "Porque eu tenho que estudar isso?", o que é reforçado pelos construtos R1, R3 e R5). Mas, segundo Keller (1987), a sensação de relevância pode vir do modo de ensinar, satisfazendo certas necessidades pessoais, como dar a pessoas com "necessidade de realização"a oportunidade de estabelecer metas e assumir a responsabilidade de alcançá-las. Neste sentido, Scratch foi positivo, considerando os construtos R6 e R7.

A satisfação está relacionada com as demais categorias, principalmente com a 
relevância percebida. As práticas de ensino devem contribuir para que os estudantes se sintam bem com suas realizações, como ao finalizar as atividades com êxito (R6).

\section{Python + JES}

A utilização de Python no contexto de mídias, apoiado pela ferramenta JES, também foi pensada para oferecer um contexto mais motivador, mais próximo da realidade dos jovens. No entanto, os resultados dos construtos de atenção e confiança levam a crer que, embora as primeiras aulas tenham sido interessantes e mantido a atenção dos estudantes, os conceitos não foram de fácil compreensão. Além disso, a maioria dos estudantes avaliaram negativamente a organização do conteúdo e a quantidade de conceitos abordados, o que leva a crer que houve uma transição brusca na complexidade do conteúdo, contribuindo para diminuir a motivação.

A relevância percebida em termos de utilidade prática foi baixa, o que, de certa forma, era esperado, já que a escolha da ferramenta e definição da abordagem consideraram a relevância em termos de "necessidade pessoal". Mas isto também não foi percebido pelos estudantes, já que a maioria deles não conseguiu relacionar o material visto com sua vida ou com conhecimentos prévios.

A maioria dos estudantes não concordaram que o JES é uma ferramenta que auxilia os novatos em programação. No entanto, consideraram importante finalizar as atividades e, em sua maioria, se sentiram bem ao completar as atividades com êxito, o que gerou percepções contraditórias sobre satisfação.

Alguns fatores externos podem ter influenciado os resultados para a terceira unidade. Uma das possíveis causas foi o desgaste sofrido devido a uma greve estudantil na unidade que o JES foi utilizado, provocando uma sensação de descontinuidade. Um outro aspecto que pode ter influenciado foi o idioma da ferramenta. Finalmente, houve mudança de professor da turma teórica na terceira unidade, o que pode também ter comprometido o processo pedagógico. Mas apesar da avaliação negativa, nenhum estudante abandonou a disciplina na terceira unidade.

\section{Conclusão}

Este artigo apresenta os resultados de um estudo que analisa a motivação de estudantes non-majors calouros que cursaram uma disciplina de Introdução a Ciência da Computação, utilizando uma abordagem adaptada para este fim, com o ambiente Scratch e com a linguagem Python combinada com desenho de figuras e manipulação de imagens.

Os resultados mostram que o ambiente Scratch pode proporcionar o aumento da motivação dos estudantes, contribuindo para seu aprendizado, realçando seu potencial para ensinar os conceitos iniciais de programação. Todavia, com a ferramenta JES no contexto de imagens, os resultados preliminares são diferentes, sugerindo que o JES não conseguiu motivar os estudantes satisfatoriamente. Este resultado pode ser devido ao ambiente, ou pode ser fruto de um conjunto de complicações ocorridos na terceira unidade da disciplina, o que demanda uma investigação adicional posterior.

Outros resultados apontam para um conjunto de variáveis que podem influenciar diretamente na motivação, contribuindo para despertá-la, mantê-la ou diminui-la, conforme apresentado em detalhes na discussão. Estes resultados são relevantes, pois fornecem indicativos de estratégias que professores podem utilizar na promoção da motivação, 
ou mesmo para evitar a sua diminuição.

Pretendemos, em breve, realizar um outro estudo com non-majors, utilizando uma versão melhorada da abordagem, bem como instrumentos de coleta de dados revisados.

\section{Referências}

Creswell, J. W. (2010). Projeto de pesquisa métodos qualitativo, quantitativo e misto. In Projeto de pesquisa métodos qualitativo, quantitativo e misto. Artmed.

da Silva, T. S. C., Tedesco, P., and de Melo, J. C. B. (2014). A importância da motivação dos estudantes e o uso de técnicas de engajamento para apoiar a escolha de jogos no ensino de programação. In Simpósio Brasileiro de Informática na Educação.

Forte, A. and Guzdial, M. (2005). Motivation and nonmajors in computer science: identifying discrete audiences for introductory courses. IEEE Transactions on Education, 48(2):248-253.

Guilloteaux, M. J. and Dörnyei, Z. (2008). Motivating language learners: A classroomoriented investigation of the effects of motivational strategies on student motivation. TESOL quarterly, pages 55-77.

Hamada, M. (2008). An integrated virtual environment for active and collaborative elearning in theory of computation. IEEE Transactions on Learning Technologies, 1(2):117-130.

Jenkins, T. (2001). Teaching programming-a journey from teacher to motivator. In The 2nd Annual Conference of the LSTN Center for Information and Computer Science.

Keller, J. M. (1987). Development and use of the ARCS model of instructional design. Journal of instructional development, 10(3):2-10.

Keller, J. M. (1993). Motivation by design. Unpublished manuscript, Florida State University, Florida.

Pears, A., Seidman, S., Malmi, L., Mannila, L., Adams, E., Bennedsen, J., Devlin, M., and Paterson, J. (2007). A Survey of Literature on the Teaching of Introductory Programming. In ITiCSE-WGR '07, pages 204-223, New York, NY, USA. ACM.

Peixoto, M. M., Scaico, P., Souza, F. V. C., and Peixoto, H. M. (2013). Uso de Estratégias de Aprendizagem e Motivacionais pelos Alunos em Disciplinas de Programação: Um Estudo de Caso na Licenciatura em Computação. In Anais do CSBC - XXI WEI.

Robins, A., Rountree, J., and Rountree, N. (2003). Learning and teaching programming: A review and discussion. Computer Science Education, 13(2):137-172.

Salazar, R., Odakura, V., and Barvinski, C. (2015). Scratch no ensino superior: motivação. In Simpósio Brasileiro de Informática na Educação-SBIE, page 1293.

Watson, C. and Li, F. W. B. (2014). Failure Rates in Introductory Programming Revisited. In Proceedings of the ITiCSE 2014, pages 39-44, New York, NY, USA. ACM.

Wlodkowski, R. J. (1978). Motivation and teaching: A practical guide. ERIC.

Yacob, A. and Saman, M. Y. M. (2012). Assessing level of motivation in learning programming among engineering students. In The International Conference on Informatics and Applications (ICIA2012), pages 425-432. 